\title{
Optimising human hepatocyte models for metabolic phenotype: effects of treatment with dimethyl sulfoxide (DMSO)
}

\author{
Nikolaos Nikolaou*, Charlotte J Green, Pippa J Gunn, Leanne Hodson, Jeremy W Tomlinson \\ University of Oxford, Oxford Centre for Diabetes, Endocrinology and Metabolism, United Kingdom
}

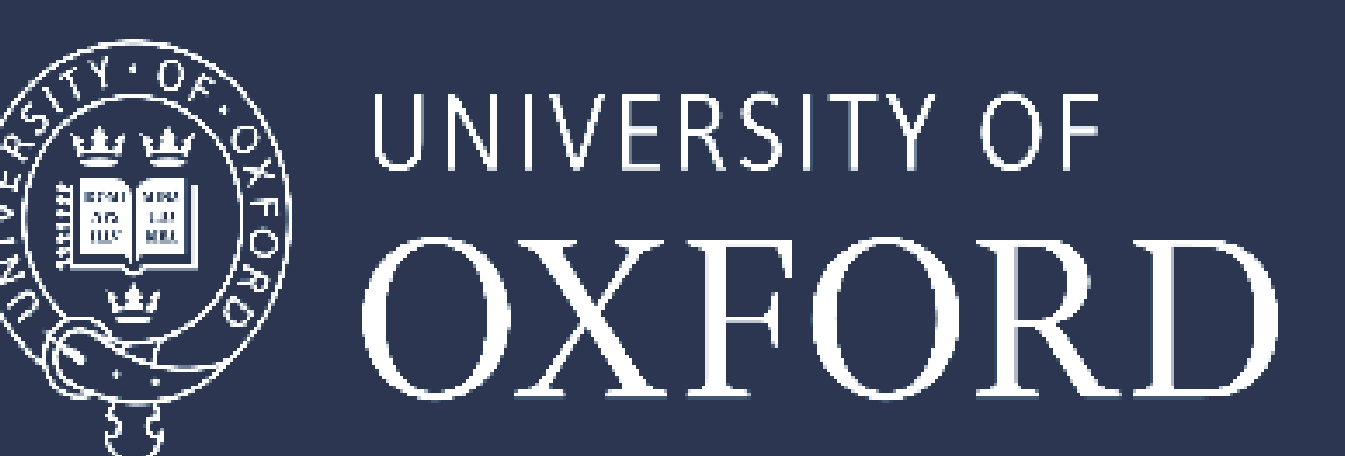

*E-mail address: nikolaos.nikolaou@keble.ox.ac.uk

Radcliffe Department of

\section{Background \& Aims}

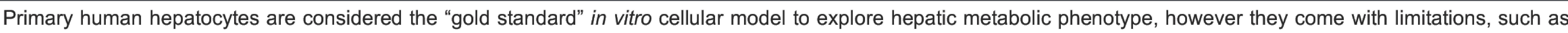

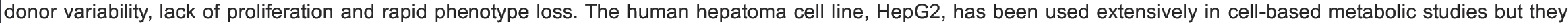

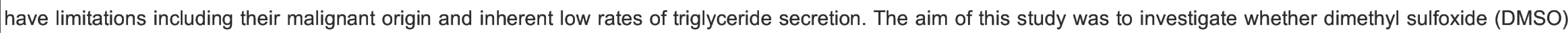

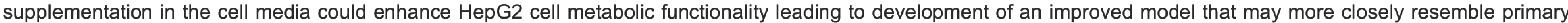
human hepatocytes.

\section{Methods}

HepG2 cells were cultured in cell media containing $1 \%$ DMSO for 7,14 and 21 days and gene expression, protein levels, intracellular triglyceride content and triglyceride, urea and 3-hydroxybutyrate concentrations in the cell medium were measured.

\section{Results}

mRNA expression of 4 markers of hepatocyte liver function (albumin, HNF4A, transthyretin and a1-antithrypsin) changed with DMSO treatment to levels that were similar to those seen in primary cultures of human hepatocytes. (Figure 1a-1d)

mRNA expression of the tumor marker alpha-fetoprotein decreased, suggesting a less malignant phenotype. ER stress marker expression (CHOP, IRE) was also decreased, as shown by western blotting experiments. (Figure 1e,1f)

DMSO treatment decreased intracellular triglyceride content while media triglyceride and 3-hydroxybutyrate levels increased in a time-dependent manner. (Figure 2) DMSO also significantly decreased the mRNA expression of genes involved in lipid metabolism (ACC1, ACC2, DGAT1, DGAT2, FAS, SCD). (Figure 3)

DMSO treatment significantly increased the mRNA expression of genes involved in glucose metabolism (PEPCK, G6PC). Changes in mRNA expression were mirrored by changes at the protein level as measured by western blotting experiments. (Figure 4)

\section{Liver function and ER stress markers}
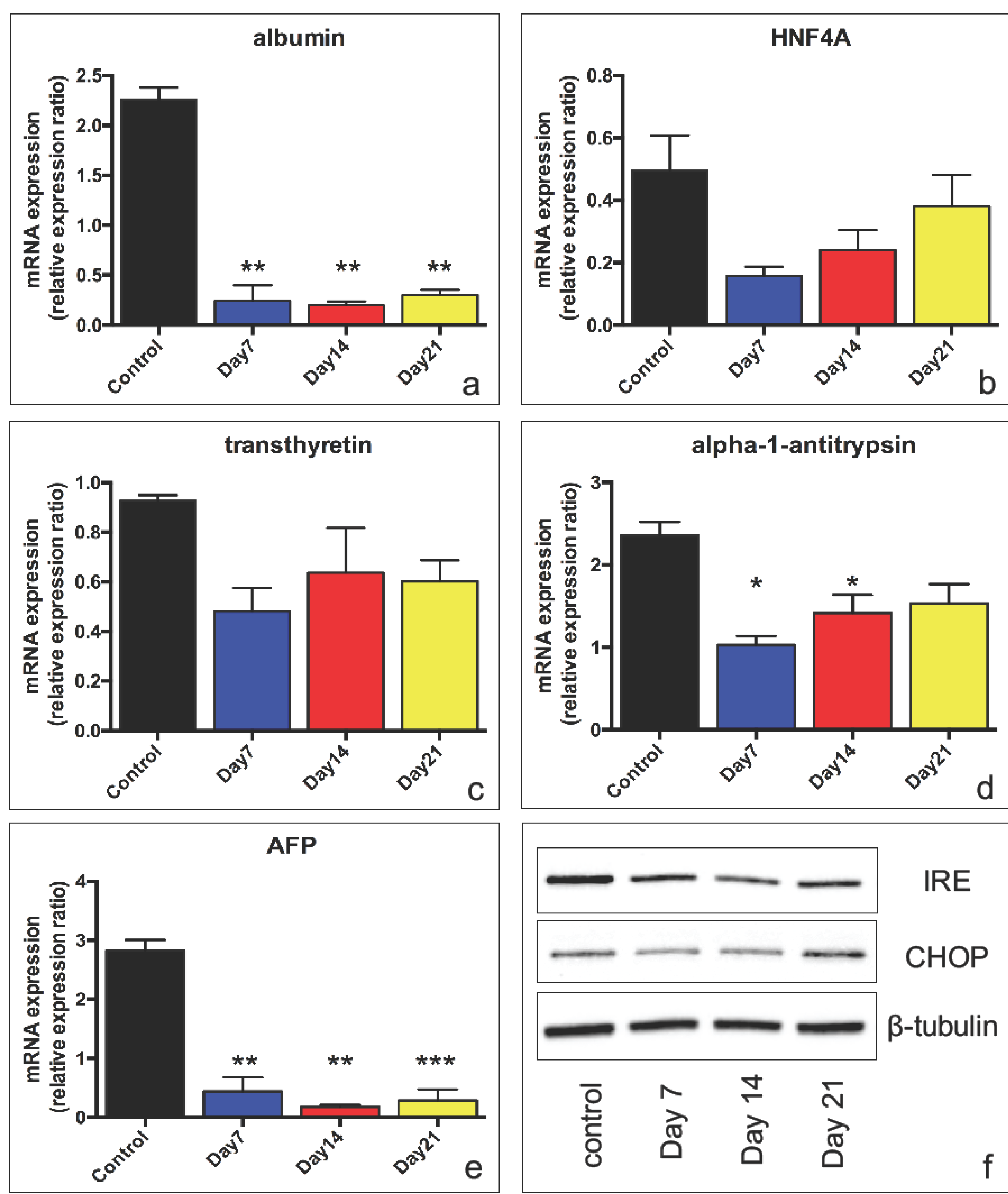

Figure 1: $m R N A$ expression of the hepatic differentiation markers a. albumin, $b$. HNF4A, c. transthyretin and d. a1-antithrypsin were decreased with DMSO treatment to levels that were similar to those from primary human hepatocytes. Also, AFP mRNA expression (e) was decreased, as well as the protein levels of the ER stress markers IRE and CHOP(f), suggesting a less malignant phenotype.

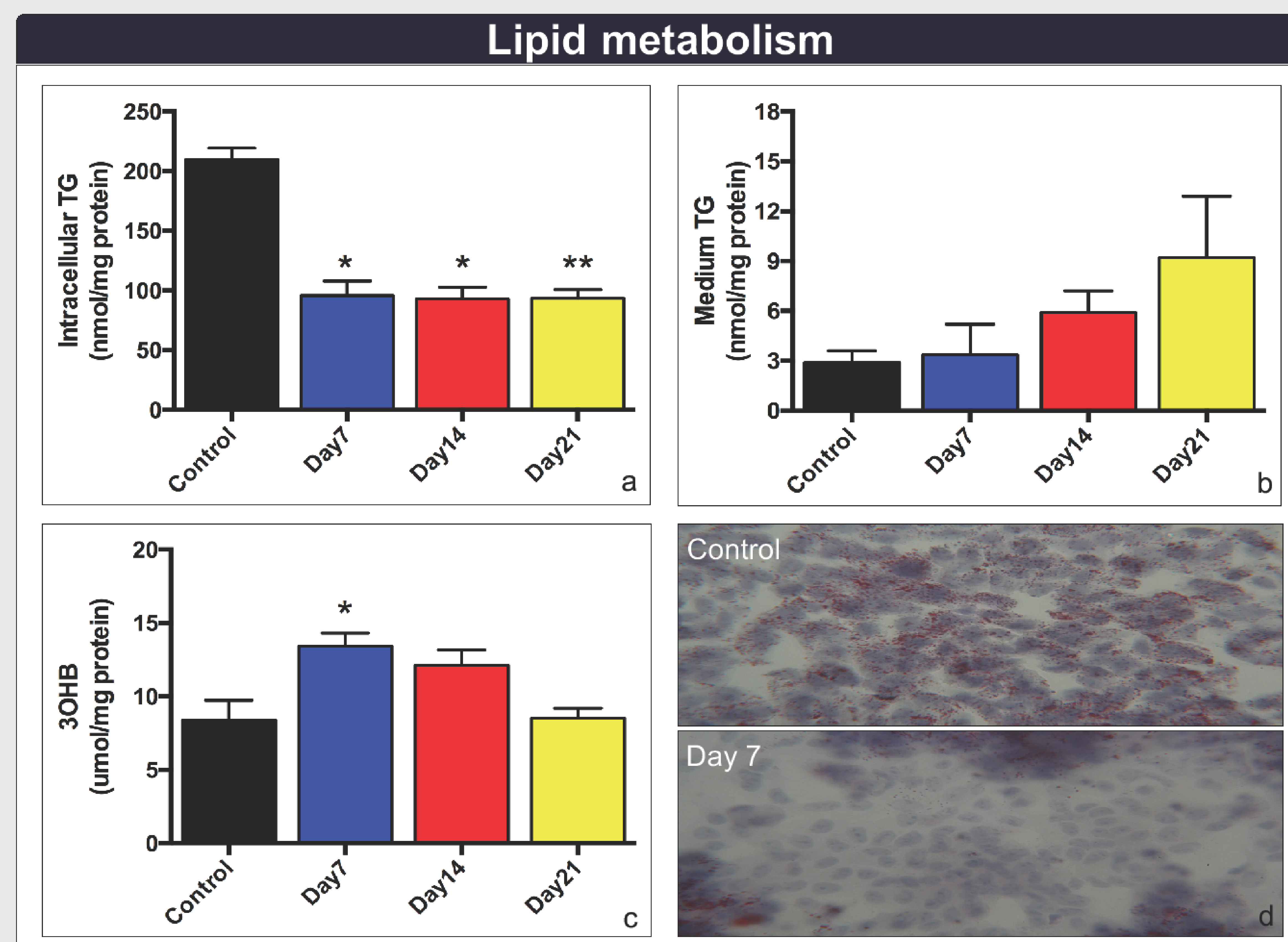

Figure 2: DMSO treatment decreased intracellular triglyceride content $(a, d)$ and increased medium triglyceride (b) as well as hydroxybutyrate levels (c) in a time-dependent manner.

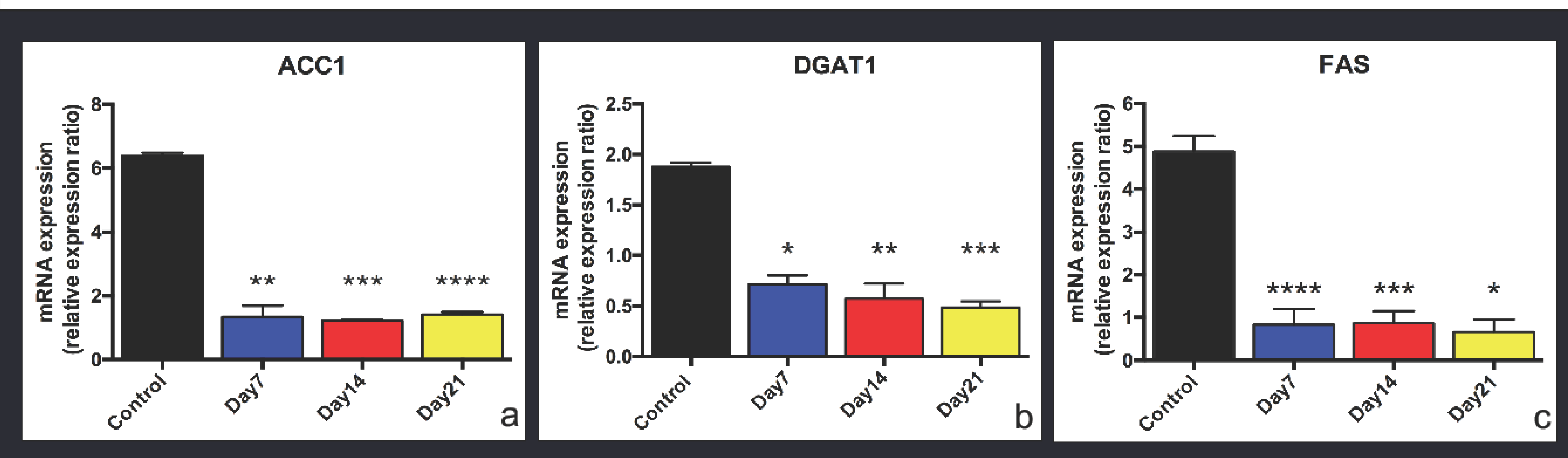

Figure 3: DMSO treatment decreased the mRNA expression of genes involved in lipid metabolism: a. ACC1, b. DGAT1 and c. FAS, in a time-dependent manner, as measured by $q P C R$

\section{Glucose metabolism}

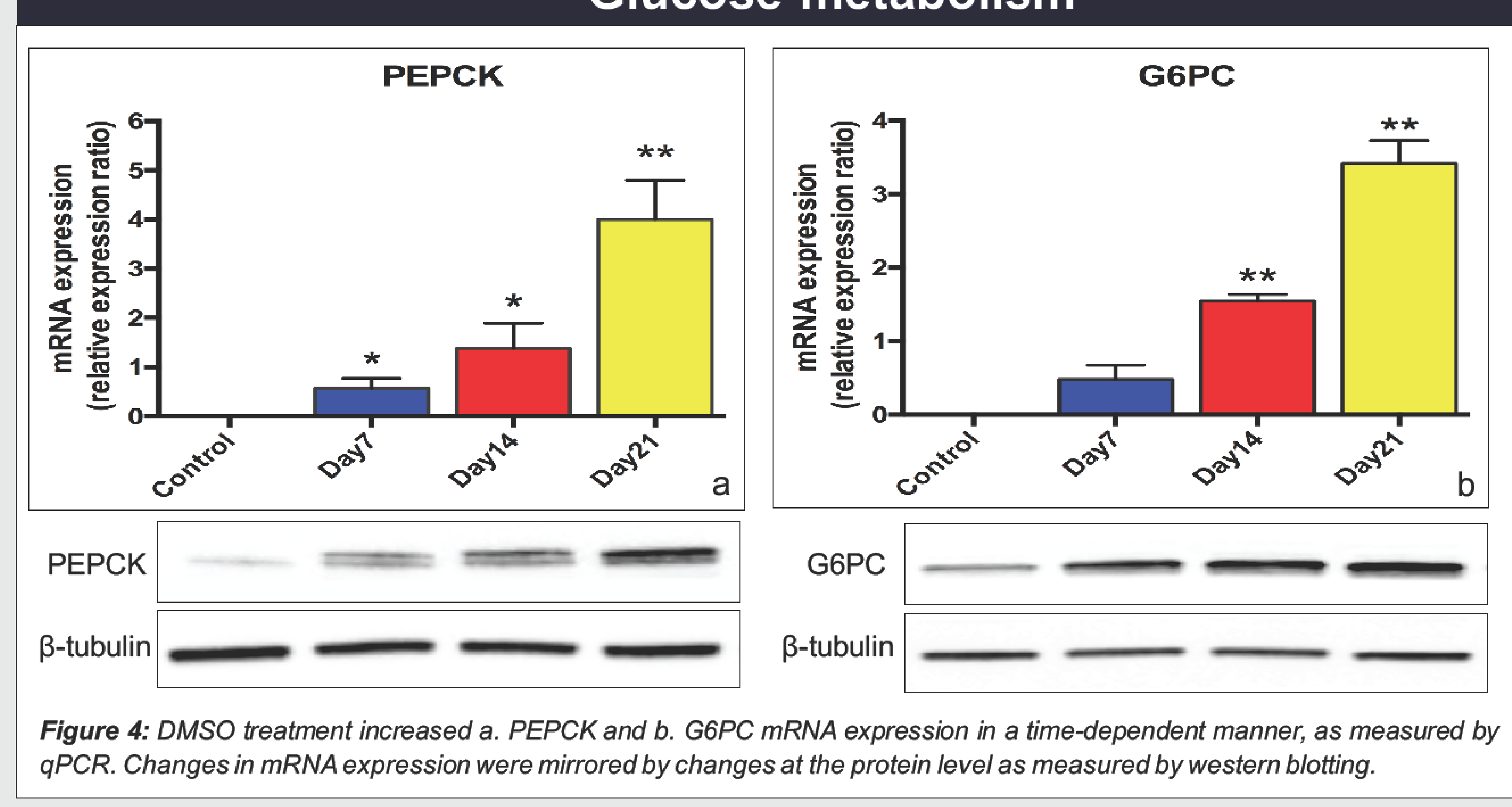

\section{Conclusion}

Our data demonstrate that DMSO treatment changes the metabolic phenotype of HepG2 cells such that they more closely resemble primary human hepatocytes and has the potential to significantly enhance currently available cell systems to study liver biology. 